\title{
Does ASTRAL score at hospital admission predict symptomatic haemorrhagic transformation in acute ischaemic stroke after revascularisation? A pilot single-centre study
}

\author{
Milena Świtońska ${ }^{1}$, Artur Słomka², Natalia Piekuś-Słomka³, Ewa Żekanowska², Grzegorz Meder \\ Paweł Sokal ${ }^{1}$, Simona Lattanzi ${ }^{5}$ \\ ${ }^{1}$ Department of Neurosurgery and Neurology, Nicolaus Copernicus University in Torun, Ludwik Rydygier Collegium Medicum, \\ Faculty of Health Sciences, Bydgoszcz, Poland \\ ${ }^{2}$ Department of Pathophysiology, Nicolaus Copernicus University in Torun, Ludwik Rydygier Collegium Medicum, \\ Faculty of Pharmacy, Bydgoszcz, Poland \\ ${ }^{3}$ Department of Inorganic and Analytical Chemistry, Nicolaus Copernicus University in Torun, Ludwik Rydygier Collegium Medicum, \\ Faculty of Pharmacy, Bydgoszcz, Poland \\ ${ }^{4}$ Department of Interventional Radiology, Jan Biziel University Hospital No. 2, Bydgoszcz, Poland \\ ${ }^{5}$ Neurological Clinic, Department of Experimental and Clinical Medicine, Marche Polytechnic University, Ancona, Italy
}

\section{ABSTRACT}

Introduction. Accurately predicting outcomes after acute ischaemic stroke (AIS) is a major clinical goal. The aim of this pilot study was to evaluate the prognostic validity and accuracy of the Acute Stroke Registry and Analysis of Lausanne (ASTRAL) score in predicting symptomatic haemorrhagic transformation $(\mathrm{sHT})$ in patients with AIS who have undergone revascularisation.

Material and methods. Consecutive patients hospitalised for AIS who underwent treatment with intravenous thrombolysis (IVT) and/or mechanical thrombectomy (MT) were identified, and their ASTRAL scores at hospital admission were estimated. The study endpoint was sHT within 24 hours of stroke onset. The predictive performance of the ASTRAL score was investigated through logistic regression analysis and discrimination and calibration tests.

Results. Sixty-eight AIS patients, with a median age of 69 (58-79) years, were included. sHT occurred in 20 (29.4\%) of the 68 patients. The ASTRAL score was significantly higher in patients who developed sHT compared to non-sHT patients [36 (34-38) versus 24 (17-32); $\mathrm{p}<0.001]$. The ASTRAL score was an independent predictor of $s H T$, and showed good discriminative power (area under the curve $0.88 ; 95 \%$ confidence interval, 0.789-0.965).

Conclusions and clinical implications. ASTRAL score is an independent predictor of sHT and shows high predictive accuracy in patients with AIS. Future studies are warranted to confirm these results.

Key words: acute ischaemic stroke, revascularisation, haemorrhagic transformation, ASTRAL

(Neurol Neurochir Pol 2022; 56 (2): 171-177)

\section{Introduction}

Despite a global decline in mortality from stroke, the rates of death and hospitalisation remain very high [1]. Intravenous thrombolysis (IVT) and mechanical thrombectomy (MT) are well-established treatments for acute ischaemic stroke (AIS). However, they are associated with an increased risk of bleeding, including symptomatic haemorrhagic transformation (sHT) $[2,3]$, so the early identification of patients at high risk of this complication is a major challenge in neurological care.

Address for correspondence: Artur Słomka, Department of Pathophysiology, Nicolaus Copernicus University in Torun, Ludwik Rydygier Collegium Medicum, M. Sklodowskiej-Curie 9 Str., 85-094 Bydgoszcz, Poland; e-mail: artur.slomka@cm.umk.pl

Received: 1.10.2021 Accepted: 5.01.2022] Early publication date: 14.02.2022

This article is available in open access under Creative Common Attribution-Non-Commercial-No Derivatives 4.0 International (CC BY-NC-ND 4.0) license, allowing to download articles and share them with others as long as they credit the authors and the publisher, but without permission to change them in any way or use them commercially. 
The Acute Stroke Registry and Analysis of Lausanne (ASTRAL) scoring system was initially designed by Ntaios et al. [4] to predict unfavourable 3-month outcomes in patients with cerebral infarction, defined as a modified Rankin Scale (mRS) score $>2$. The model includes the following six variables: age (A), severity of stroke assessed on admission by means of National Institutes of Health Stroke Scale (NIHSS) score (S), time from symptom onset to admission $(\mathrm{T})$, visual field range (R), acute glucose level (A), and level of consciousness (L). Interestingly, the accuracy of this scoring system to predict sHT has not previously been evaluated. In this pilot study, we have examined the role of the ASTRAL score collected at hospital admission in predicting SHT in AIS patients undergoing revascularisation.

\section{Material and methods}

We included in this study consecutive patients with AIS admitted to the Interventional Stroke Treatment Centre of University Hospital No. 2 in Bydgoszcz, Poland from January 2017 to December 2019. We did not include patients treated in 2020 or 2021 due to the possible impact of severe acute respiratory syndrome coronavirus 2 (SARS-CoV-2) infection on the variables studied. All patients were admitted within 24 hours of symptom onset. Patients with haemorrhagic stroke, transient ischaemic attack (TIA), recent myocardial infarction (MI), active infection, liver or kidney failure, cancer, and steroid therapy were excluded. We also excluded patients with procedural intracranial haemorrhage, i.e. intracerebral haemorrhage clearly associated with the procedure itself as secondary to arterial dissection or vessel perforation and subarachnoid haemorrhage. The diagnosis of AIS was made in accordance with World Health Organisation (WHO) criteria. Any stroke was classified according to the Trial of ORG 10172 in Acute Stroke Treatment (TOAST) criteria and to the Oxfordshire Community Stroke Project (OCSP) $[5,6]$. Stroke severity was quantified using the National Institutes of Health Stroke Scale (NIHSS). The extension of the ischaemic lesion was estimated based on the Alberta Stroke Programme Early CT Score (ASPECTS) on head CT performed in an emergency before causal treatment. Further details on study design and data collection have previously been published [7].

Patients with AIS received causal treatment (intravenous thrombolysis [IVT] and/or mechanical thrombectomy [MT]) following the recommendations of the American Heart Association/American Stroke Association [8]. Thrombectomy was performed by the same interventional radiologist (GM) in patients who had an occluded large cerebral artery, i.e. the middle cerebral artery (M1/M2) or basilar artery, using aspiration catheters alone, stent-retrievers alone, or both, depending on the occlusion type/location and the choice of the neuro-interventionist. The degree of recanalisation was assessed using the Thrombolysis in Cerebral Infarction (TICI) scale [9]. A follow-up CT scan was performed routinely within
24 hours from IVT and/or MT, and thereafter on an individual basis in the case of any clinical deterioration.

Symptomatic haemorrhagic transformation (sHT) was defined as parenchymal haematoma $(\mathrm{PH})>30 \%$ of the infarcted area with a significant space-occupying effect or clot remote from the infarcted area (PH2) according to the criteria of the European Cooperative Acute Stroke Study II (ECASS II) [10-12] associated with a worsening of the NIHSS score by 4 or more points within 24 hours of stroke onset [13].

The ASTRAL score has been estimated on the basis of age, stroke severity according to admission NIHSS score, time delay between symptom onset or last proof of good health (in cases of unknown onset of stroke) and admission, presence of deficits in visual field, blood glucose levels at admission, and level of consciousness [4]. The ASTRAL score was calculated for each AIS patient at hospital admission.

The normal distribution of each continuous variable was assessed using a Shapiro-Wilk test. Values were reported as medians (interquartile ranges) or the number of patients. The characteristics of patients with sHT and without sHT were compared using the Mann-Whitney $U$ test and Pearson's $\chi 2$ tests. According to Receiver Operating Characteristics Curve (ROC) analysis, the optimal cut-off value was determined as the point maximising the Youden function, which is the difference between the true positive rate and the false-positive rate over all possible cut-off point values. Results were considered significant for $\mathrm{p}$ values $<0.05$ (two-sided). STATISTICA software was used (version 13.3, TIBCO Software Inc., Palo Alto, CA, USA).

The study protocol was designed following local and international ethics criteria for human research. The Bioethical Commission at the Collegium Medicum of Nicolaus Copernicus University (Bydgoszcz, Poland) approved the study (KB 694/2016), and written informed consent was obtained from the patients or their family members. Anonymised data will be shared upon request from any qualified investigator.

\section{Results}

In the present study, 381 patients were initially screened and a total of 68 patients with AIS were selected and included according to our inclusion and exclusion criteria. The reasons for exclusion were: evidence of active infection before admission or any systemic infection that occurred during the first 48 hours after causal treatment $(\mathrm{n}=227)$; cancer, chronic inflammation, autoimmune disease, or steroid therapy $(n=30)$; and the unavailability of medical records as discharge occurred on the same day as admission $(\mathrm{n}=56)$. Recombinant tissue plasminogen activator (rtPA) was administered intravenously to 53 patients, 24 of whom also received MT. Fifteen patients underwent MT alone as they exceeded the time window (> 4.5 hours) for the IVT. Demographic and clinical characteristics of the study population are set out in Table 1, which also presents the factors associated with an unfavourable prognosis 
Table 1. Demographic and clinical characteristics of study population

\begin{tabular}{|c|c|c|c|c|c|}
\hline \multicolumn{2}{|c|}{ Parameter [unit] } & $\begin{array}{l}\text { AIS patients } \\
\qquad(\mathrm{n}=68)\end{array}$ & $\begin{array}{l}\text { Non-sHT } \\
(n=48)\end{array}$ & $\begin{array}{c}\text { sHT } \\
(n=20)\end{array}$ & P-value \\
\hline \multicolumn{2}{|l|}{ Age [years] } & $69(58-79)$ & $67(55-78)$ & $73(64-81)$ & 0.135 \\
\hline \multicolumn{2}{|l|}{ Male gender } & $34(50 \%)$ & $22(46 \%)$ & $12(60 \%)$ & 0.287 \\
\hline \multicolumn{2}{|c|}{ Body mass index (BMI), [kg/m2] } & $27(24-31)$ & $27(24-32)$ & $28(25-31)$ & 0.819 \\
\hline \multirow{7}{*}{$\begin{array}{l}\text { Medical history and } \\
\text { ischaemic stroke } \\
\text { risk factors }[n, \%]\end{array}$} & Coronary artery disease (CAD) & $18(26 \%)$ & $10(21 \%)$ & $8(40 \%)$ & 0.103 \\
\hline & Previous ischaemic stroke & $10(15 \%)$ & $7(15 \%)$ & $3(15 \%)$ & 0.965 \\
\hline & Hypertension & $50(74 \%)$ & $34(71 \%)$ & $16(80 \%)$ & 0.435 \\
\hline & Diabetes mellitus (DM) & $22(32 \%)$ & $14(29 \%)$ & $8(40 \%)$ & 0.384 \\
\hline & Dyslipidemia & $38(56 \%)$ & $29(60 \%)$ & $9(45 \%)$ & 0.243 \\
\hline & Atrial fibrillation (AF) & $24(35 \%)$ & $12(25 \%)$ & $12(60 \%)$ & 0.006 \\
\hline & Current smokers & $20(29 \%)$ & $13(27 \%)$ & $7(35 \%)$ & 0.514 \\
\hline \multirow{2}{*}{$\begin{array}{l}\text { Anticoagulant } \\
\text { therapy }[n, \%]\end{array}$} & Vitamin $\mathrm{K}$ antagonists & $8(12 \%)$ & $5(10 \%)$ & $3(15 \%)$ & 0.593 \\
\hline & Acetylsalicylic acid (ASA) & $21(31 \%)$ & $14(29 \%)$ & $7(35 \%)$ & 0.635 \\
\hline \multicolumn{2}{|c|}{ Systolic blood pressure (SBP), [mmHg] } & $140(130-160)$ & $130(130-150)$ & $155(143-180)$ & 0.001 \\
\hline \multicolumn{2}{|c|}{ Diastolic blood pressure (DBP), [mmHg] } & $80(80-90)$ & $80(80-85)$ & $90(80-100)$ & 0.001 \\
\hline \multicolumn{2}{|c|}{ Left ventricle ejection fraction (LVEF), [\%] } & $60(50-65)$ & $60(50-65)$ & $59(45-60)$ & 0.084 \\
\hline \multirow{8}{*}{$\begin{array}{l}\text { Laboratory } \\
\text { parameters }\end{array}$} & Aspartate transaminase (AST), [U/I] & $21(16-27)$ & $20(16-25)$ & $22(18-29)$ & 0.156 \\
\hline & Alanine transaminase & $17(13-25)$ & $17(13-26)$ & $17(11-21)$ & 0.436 \\
\hline & $(\mathrm{ALT}),[\mathrm{U} / \mathrm{l}]$ & & & & \\
\hline & Serum creatinine $[\mathrm{mg} / \mathrm{dL}]$ & $0.89(0.73-1.10)$ & $0.85(0.69-1.07)$ & $1.04(0.80-1.15)$ & 0.030 \\
\hline & Low-density lipoprotein (LDL), [mg/dL] & $86(72-119)$ & $91(74-135)$ & $76(67-93)$ & 0.071 \\
\hline & Glucose [mg/dL] & $128(107-160)$ & $122(106-151)$ & $140(108-198)$ & 0.146 \\
\hline & International normalised ratio (INR) & $1.1(1.0-1.2)$ & $1.1(1.0-1.2)$ & $1.2(1.1-1.4)$ & 0.006 \\
\hline & Activated partial thromboplastin time (aPTT), [s] & $26.5(25.1-29.4)$ & $26.2(24.5-29.5)$ & $27.5(25.8-29.2)$ & 0.279 \\
\hline \multirow{5}{*}{$\begin{array}{l}\text { Trial of ORG } 10172 \\
\text { in Acute Stroke } \\
\text { Treatment (TOAST) } \\
\text { classification [n, \%] }\end{array}$} & Large-artery atherosclerosis (LAA) & $13(19 \%)$ & $8(17 \%)$ & $5(25 \%)$ & 0.003 \\
\hline & Small vessel occlusion (SVO) & $5(7 \%)$ & $5(10 \%)$ & 0 & \\
\hline & Cardioembolism (CE) & $27(40 \%)$ & $13(27 \%)$ & $14(70 \%)$ & \\
\hline & Stroke of other determined aetiology (SOA) & $3(4 \%)$ & $3(6 \%)$ & 0 & \\
\hline & Stroke of undetermined aetiology (SUA) & $20(29 \%)$ & $19(40 \%)$ & $1(5 \%)$ & \\
\hline \multirow{4}{*}{$\begin{array}{l}\text { Oxfordshire } \\
\text { Community Stroke } \\
\text { Project (OCSP) } \\
\text { classification [n, \%] }\end{array}$} & Lacunar infarct (LACI) & $13(19 \%)$ & $13(27 \%)$ & 0 & 0.001 \\
\hline & Partial anterior circulation infarct (PACI) & $29(43 \%)$ & $22(46 \%)$ & $7(35 \%)$ & \\
\hline & Total anterior circulation infarct (TACl) & $17(25 \%)$ & $6(13 \%)$ & $11(55 \%)$ & \\
\hline & Posterior circulation infarct (POCI) & $9(13 \%)$ & $7(15 \%)$ & $2(10 \%)$ & \\
\hline \multirow{3}{*}{$\begin{array}{l}\text { Type of therapy } \\
{[n, \%]}\end{array}$} & Thrombolysis (IVT) & $29(43 \%)$ & $28(58 \%)$ & $1(5 \%)$ & 0.00004 \\
\hline & $\begin{array}{l}\text { Thrombolysis (IVT) and mechanical } \\
\text { thrombectomy (MT) }\end{array}$ & $24(35 \%)$ & $15(31 \%)$ & $9(45 \%)$ & \\
\hline & Mechanical thrombectomy (MT) & $15(22 \%)$ & $5(10 \%)$ & $10(50 \%)$ & \\
\hline \multirow[t]{3}{*}{ Stroke scales } & $\begin{array}{l}\text { Acute Stroke Registry and Analysis of Lausanne } \\
\text { (ASTRAL) }\end{array}$ & $29(22-35)$ & $24(17-32)$ & $36(34-38)$ & 0.000001 \\
\hline & National Institutes of Health Stroke Scale (NIHSS) & $13(7-17)$ & $10(6-15)$ & $17(15-21)$ & 0.0003 \\
\hline & $\begin{array}{l}\text { Alberta Stroke Programme Early CT Score } \\
\text { (ASPECTS) }\end{array}$ & $9(9-10)$ & $10(8-10)$ & $9(9-10)$ & 0.938 \\
\hline \multirow{4}{*}{$\begin{array}{l}\text { Thrombolysis in } \\
\text { Cerebral Infarction } \\
\text { (TICI) scale [n, \%] }\end{array}$} & $\mathrm{TIClO}$ & $4(11 \%)$ & $3(15 \%)$ & $1(5 \%)$ & 0.364 \\
\hline & $\mathrm{TICl} 2 \mathrm{a}$ & $1(3 \%)$ & 0 & $1(5 \%)$ & \\
\hline & $\mathrm{TICl} 2 \mathrm{~b}$ & $11(28 \%)$ & $7(35 \%)$ & $4(21 \%)$ & \\
\hline & $\mathrm{TICl} 3$ & $23(59 \%)$ & $10(50 \%)$ & $13(68 \%)$ & \\
\hline
\end{tabular}


following IS [14]. The study population consisted of 34 males and 34 females, with a median age of $69(58-79)$ years. A total of 20 out of 68 (29.4\%) patients experienced sHT. Our cohort was then divided into two groups depending on the presence or absence of sHT (Table 1). The two groups did not significantly differ in most clinical and laboratory characteristics, except that the sHT group tended to have a higher NIHSS, more elevated serum creatinine levels, and a greater international normalised ratio (INR) than the non-sHT group. Additionally, patients from the sHT group were more likely to have AF and higher blood pressure, but not other risk factors. The two groups also differed in the TOAST and OCSP classification and the type of therapy.

The median ASTRAL score at admission was 29 (22-35). Symptomatic haemorrhagic transformation ( $\mathrm{sHT}$ ) within 24 hours of stroke onset was diagnosed in 20 (29.4\%) patients, of whom one underwent IVT alone, nine underwent IVT plus MT, and 10 underwent MT alone. The ASTRAL score was significantly higher in patients who developed sHT compared to non-sHT patients [36 (34-38) vs. 24 (17-32); p < 0.001, Figure $1 \mathrm{~A}]$. In ROC analysis with respect to $\mathrm{sHT}$, the area under the curve (AUC) was 0.877 (95\% CI, 0.789-0.965; SE, 0.045; Figure 1B). The Youden's index identified 35 to be the best cut-off of the ASTRAL score to discriminate patients experiencing sHT, yielding a sensitivity of $75 \%$ and a specificity of $92 \%$.

\section{Discussion}

This pilot study showed that the ASTRAL score can predict with good discriminative power sHT in AIS patients undergoing revascularisation. Patients who developed sHT after revascularisation had significantly higher ASTRAL values compared to patients with AIS and no sHT. Furthermore, a score of 35 on the ASTRAL scale was the optimal threshold to predict haemorrhagic transformation of a brain infarct.

Developed from the ASTRAL registry as an integer-based score to predict 3-month functional status in AIS [4], the ASTRAL score has been externally validated in AIS patients undergoing IVT [15], and in patients with embolic strokes of undetermined source [16]. This scoring system has also shown remarkable consistency in predicting long-term functional outcomes and mortality in unselected AIS patients, both in European and non-European populations [17-20]. Interestingly, the ASTRAL score has been demonstrated to predict outcomes of AIS patients with greater accuracy than physicians. In an online anonymous survey, 244 physicians interested in stroke from 32 different countries provided outcome estimates in randomly allocated structured scenarios of recent real-life stroke patients: $56.8 \%$ of the physicians' estimates about the percentage probability of 3-month mRS $>2$ were accurate, compared to $86.5 \%$ of the ASTRAL score estimates [21].

Clinical grading systems can be useful tools to stratify patient risk and estimate prognosis, to help counsel patients and their families, and to standardise communication among healthcare providers. As practical tools to promote information, scoring models need to be easily computed and widely applicable in clinical practice. In this regard, the ASTRAL score contains readily accessible parameters, does not require imaging data or complex mathematical algorithms, and can be used early in an emergency setting. The availability of a scoring system that is easy to perform and interpret in everyday clinical practice, and is able to predict sHT, appears to be extremely important considering that IS patient management differs
A

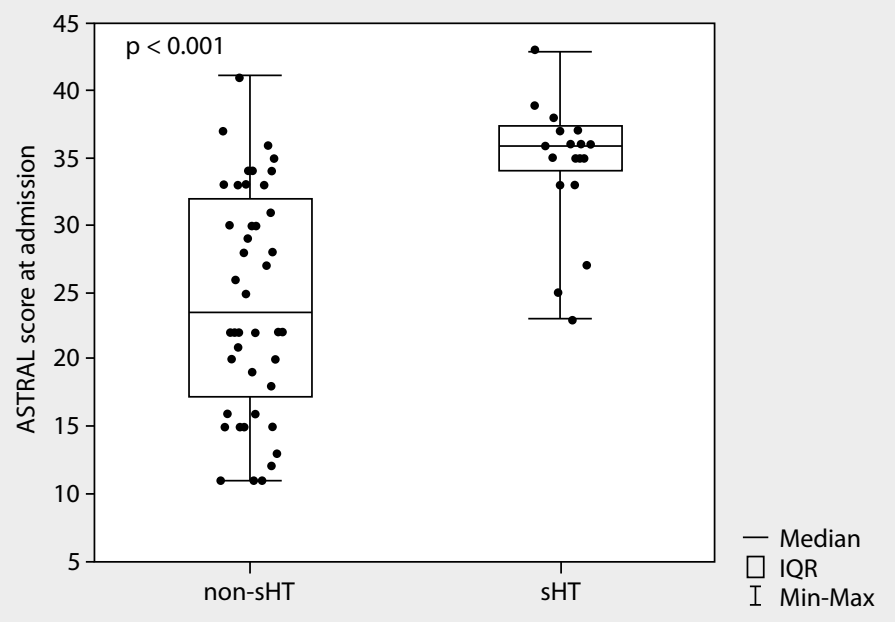

B

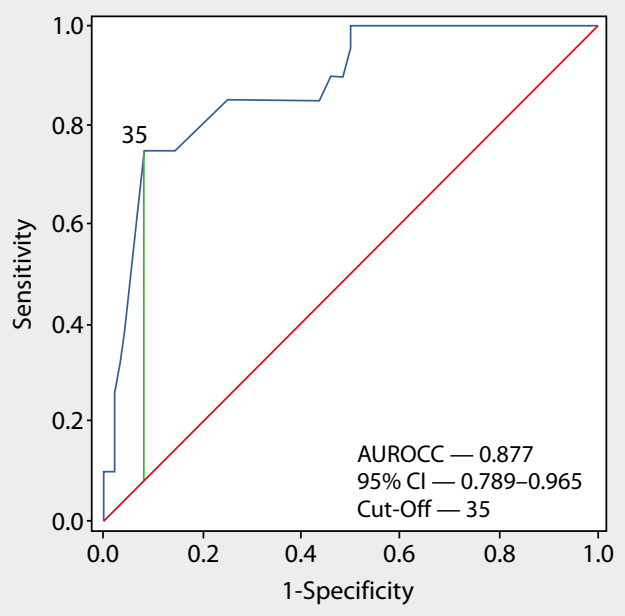

Figure 1. Comparison of Acute Stroke Registry and Analysis of Lausanne (ASTRAL) scores between symptomatic haemorrhagic transformation (sHT) patients and non-sHT patients $(\mathbf{A})$. Receiver operating characteristics analysis for prediction of sHT in acute ischaemic stroke (AIS) patients undergoing revascularisation (B) 
significantly depending on the region of Poland [22]. To the best of our knowledge, this is the first study to highlight the potential role of the ASTRAL score as a predictor of sHT in patients treated with IVT and/or MT. The previous study by Asuzu et al. [23] did not find any association between the ASTRAL score and sHT. Differences between the studies may help explain these discrepant findings. It is worth noting that in the earlier study, a different definition of sHT was adopted (i.e. National Institute of Neurological Diseases and Stroke (NINDS) vs. ECASS II) [23]. Also, although the demographic and clinical characteristics of patients included in the two studies were overall similar, differences existed in the treatment strategies, as patients included in the Asuzu et al. study underwent treatment with IVT only. Further studies are needed to fully explore how acute stroke treatment may influence the predictive role of the ASTRAL score. Other scales with potential use in sHT prediction include the DRAGON, StrokeTPI (stroke-thrombolytic predictive instrument), and HAT (haemorrhage after thrombolysis) scores, among others [23]. Promising observations have also been made with the TURN (thrombolysis risk using mRS and NIHSS) score, which, due to its simplicity, may be an excellent tool in predicting sHT, but requires validation in IS patients after MT [24, 25]. Another indicator with a proven high predictive value of HT after IS is the HTI (haemorrhagic transformation index), regardless of the use of IV rt-PA [26]. However, the HTI is an indicator with a rather complex structure, being composed of ASPECTS, NIHSS, hyperdense middle cerebral artery (HMCA) sign, and the presence of AF on ECG at admission. This complexity limits its use in neurological practice.

An essential element of our study is the high rate of sTH observed in patients with AIS. Many factors can influence the onset of sHT [27], and well-known factors include, but are not limited to, stroke severity, reperfusion therapy, hypertension, hyperglycaemia, and age [28]. Factors that significantly increase the risk of $\mathrm{sHT}$ in patients undergoing revascularisation treatment are also poor collateral status and variables related to the MT procedure $[29,30]$.

The patients included in our study who developed sHT had a relatively severe course of AIS, frequent incidence of $\mathrm{AF}$, and high blood pressure, all of which are associated with sHT $[2,27]$. Moreover, $70 \%$ of patients in the sHT group were diagnosed with cardioembolic (CE) stroke, a crucial aetiological component of sHT [30]. All these factors may have contributed to the high incidence of sHT observed in our study, which undoubtedly requires further detailed analysis.

As a pilot research study, it may be considered reliable for the development of preliminary insights and for suggesting working hypotheses. Some shortcomings need to be considered in the interpretation of the findings, such as the small sample size and the inherent limits of any single-centre retrospective analysis, e.g. selection and collection biases resulting from residual confounding due to unmeasured or incompletely characterised covariates. Future studies, if possible prospective multicentre cohort studies, should also include in the statistical analysis other factors mentioned in the previous paragraph that may influence the occurrence of sHT.

In this study, due to the relatively small sample size, such results have not been presented. Our research also lacks data on the technical aspects of MT due to the pilot nature of this treatment programme in the clinic. These aspects should be considered in subsequent investigations, especially in the light of recent studies showing that the degree of recanalisation after MT depends on the time elapsed from stroke onset to groin puncture [31]. However, these procedural characteristics do not seem to affect the ASTRAL value itself, which is assessed at admission to the hospital, i.e. before any causal treatment of AIS has been performed. Our study's main strengths include the use of widely accessible variables and the cost-effectiveness of the ASTRAL score. Furthermore, low heterogeneity existed in baseline patient characteristics, because all patients had no pre-morbid neurological dysfunctions.

Previously, various clinical and laboratory parameters have been proposed to predict the haemorrhagic transformation of AIS. These parameters, including age and well-defined ischaemic stroke comorbidities, have been characterised by a relatively high variability and inconsistent sensitivity in determining the risk of HT $[32,33]$. Furthermore, the predictive models are usually based on assessing several parameters simultaneously, which may hinder decision-making in rapid neurological diagnosis [34]. Specific laboratory parameters, such as the measurement of blood caveolin-1 and caveolin-2, are not routinely used, and the laboratory methods used to determine their levels have not been sufficiently validated $[33,35]$.

Therefore, our research suggests a high clinical utility of the ASTRAL score to predict sHT after the causative treatment of AIS subjects. Our study also provides an incentive for further studies assessing the possibility of combining ASTRAL and other parameters for sHT risk assessment.

\section{Conclusions and clinical implications}

In summary, the ASTRAL score can predict symptomatic haemorrhagic transformation in AIS patients undergoing reperfusion therapies. Its good discriminatory ability may widen the applicability and broaden the potentiality of this tool as a reliable instrument in clinical practice and stroke research. Further investigations are warranted to validate these encouraging findings in larger independent cohorts of patients treated with thrombectomy, or thrombolysis combined with thrombectomy.

Conflicts of interest: None.

Funding: None.

\section{References}

1. GBD 2016 Stroke Collaborators. Global, regional, and national burden of stroke, 1990-2016: a systematic analysis for the Global Burden 
of Disease Study 2016. Lancet Neurol. 2019; 18(5): 439-458, doi: 10.1016/S1474-4422(19)30034-1, indexed in Pubmed: 30871944.

2. Yaghi S, Willey JZ, Cucchiara B, et al. American Heart Association Stroke Council; Council on Cardiovascular and Stroke Nursing; Council on Clinical Cardiology; and Council on Quality of Care and Outcomes Research. Treatment and Outcome of Hemorrhagic Transformation After Intravenous Alteplase in Acute Ischemic Stroke: A Scientific Statement for Healthcare Professionals From the American Heart Association/American Stroke Association. Stroke. 2017; 48(12): e343e361, doi: 10.1161/STR.0000000000000152, indexed in Pubmed: 29097489.

3. Zhang X, Xie Yi, Wang H, et al. Symptomatic Intracranial Hemorrhage After Mechanical Thrombectomy in Chinese Ischemic Stroke Patients: The ASIAN Score. Stroke. 2020; 51(9): 2690-2696, doi: 10.1161/ STROKEAHA.120.030173, indexed in Pubmed: 32811387.

4. Ntaios G, Faouzi M, Ferrari J, et al. An integer-based score to predict functional outcome in acute ischemic stroke: the ASTRAL score. Neurology. 2012; 78(24): 1916-1922, doi: 10.1212/ WNL.0b013e318259e221, indexed in Pubmed: 22649218.

5. Adams HP, Bendixen BH, Kappelle $\sqcup$, et al. Classification of subtype of acute ischemic stroke. Definitions for use in a multicenter clinical trial. TOAST. Trial of Org 10172 in Acute Stroke Treatment. Stroke. 1993; 24(1): 35-41, doi: 10.1161/01.str.24.1.35, indexed in Pubmed: 7678184.

6. Bamford J, Sandercock P, Dennis M, et al. Classification and natural history of clinically identifiable subtypes of cerebral infarction. Lancet. 1991; 337(8756): 1521-1526, doi: 10.1016/0140-6736(91)93206o, indexed in Pubmed: 1675378.

7. Świtońska M, Piekuś-Słomka N, Słomka A, et al. Neutrophil-to-Lymphocyte Ratio and Symptomatic Hemorrhagic Transformation in Ischemic Stroke Patients Undergoing Revascularization. Brain Sci. 2020; 10(11), doi: 10.3390/brainsci10110771, indexed in Pubmed: 33114150.

8. Powers WJ, Derdeyn CP, Biller J, et al. American Heart Association Stroke Council. 2015 American Heart Association/American Stroke Association Focused Update of the 2013 Guidelines for the Early Management of Patients With Acute Ischemic Stroke Regarding Endovascular Treatment: A Guideline for Healthcare Professionals From the American Heart Association/American Stroke Association. Stroke. 2015; 46(10): 3020-3035, doi: 10.1161/STR.0000000000000074, indexed in Pubmed: 26123479.

9. Higashida R, Furlan A. Technology Assessment Committees of the American Society of Interventional and Therapeutic Neuroradiology and the Society of Interventional Radiology. Trial Design and Reporting Standards for Intra-Arterial Cerebral Thrombolysis for Acute Ischemic Stroke. Stroke. 2003; 34(8): 109-137, doi: 10.1161/01. str.0000082721.62796.09.

10. Hacke W, Kaste M, Fieschi C, et al. Randomised double-blind placebocontrolled trial of thrombolytic therapy with intravenous alteplase in acute ischaemic stroke (ECASS II). Second European-Australasian Acute Stroke Study Investigators. Lancet. 1998; 352(9136): 1245-1251, doi: 10.1016/s0140-6736(98)08020-9, indexed in Pubmed: 9788453.

11. Nogueira RG, Gupta R, Jovin TG, et al. Predictors and clinical relevance of hemorrhagic transformation after endovascular therapy for anterior circulation large vessel occlusion strokes: a multicenter retrospective analysis of 1122 patients. J Neurointerv Surg. 2015; 7(1): 16-21, doi: 10.1136/neurintsurg-2013-010743, indexed in Pubmed: 24401478.

12. Fiorelli M, Bastianello S, von Kummer R, et al. Hemorrhagic transformation within 36 hours of a cerebral infarct: relationships with early clinical deterioration and 3-month outcome in the European Cooperative Acute Stroke Study I (ECASS I) cohort. Stroke. 1999; 30(11): 2280-2284, doi: 10.1161/01.str.30.11.2280, indexed in Pubmed: 10548658.

13. Saver JL, Jahan R, Levy El, et al. SWIFT Trialists. Solitaire flow restoration device versus the Merci Retriever in patients with acute ischaemic stroke (SWIFT): a randomised, parallel-group, non-inferiority trial. Lancet. 2012; 380(9849): 1241-1249, doi: 10.1016/S01406736(12)61384-1, indexed in Pubmed: 22932715.

14. Wańkowicz P, Gołąb-Janowska M, Nowacki P. Risk factors for death by acute ischaemic stroke in patients from West-Pomerania, Poland. Neurol Neurochir Pol. 2020; 54(2): 150-155, doi: 10.5603/PJNNS. a2020.0018, indexed in Pubmed: 32101324.

15. Cooray C, Mazya M, Bottai M, et al. External Validation of the ASTRAL and DRAGON Scores for Prediction of Functional Outcome in Stroke. Stroke. 2016; 47(6): 1493-1499, doi: 10.1161/STROKEAHA.116.012802, indexed in Pubmed: 27174528.

16. Lattanzi S, Pulcini A, Corradetti T, et al. Prediction of Outcome in Embolic Strokes of Undetermined Source. J Stroke Cerebrovasc Dis. 2020; 29(1): 104486, doi: 10.1016/j.jstrokecerebrovasdis.2019.104486, indexed in Pubmed: 31706753.

17. Wang WY, Sang WW, Jin Di, et al. The Prognostic Value of the iScore, the PLAN Score, and the ASTRAL Score in Acute Ischemic Stroke. J Stroke Cerebrovasc Dis. 2017; 26(6): 1233-1238, doi: 10.1016/j. jstrokecerebrovasdis.2017.01.013, indexed in Pubmed: 28236594.

18. Shen Bo, Yang Xu, Sui RB, et al. The Prognostic Value of the THRIVE Score, the iScore Score and the ASTRAL Score in Chinese Patients With Acute Ischemic Stroke. J Stroke Cerebrovasc Dis. 2018; 27(10): 2877-2886, doi: 10.1016/j.jstrokecerebrovasdis.2018.06.011, indexed in Pubmed: 30077603.

19. Liu G, Ntaios G, Zheng H, et al. External validation of the ASTRAL score to predict 3- and 12-month functional outcome in the China National Stroke Registry. Stroke. 2013; 44(5): 1443-1445, doi: 10.1161/ STROKEAHA.113.000993, indexed in Pubmed: 23493731.

20. Papavasileiou V, Milionis H, Michel P, et al. ASTRAL score predicts 5-year dependence and mortality in acute ischemic stroke. Stroke. 2013; 44(6): 1616-1620, doi: 10.1161/STROKEAHA.113.001047, indexed in Pubmed: 23559264.

21. Ntaios G, Gioulekas F, Papavasileiou V, et al. ASTRAL, DRAGON and SEDAN scores predict stroke outcome more accurately than physicians. Eur J Neurol. 2016; 23(11): 1651-1657, doi: 10.1111/ ene.13100, indexed in Pubmed: 27456206.

22. Maluchnik M, Ryglewicz D, Sienkiewicz-Jarosz $\mathrm{H}$, et al. Differences in acute ischaemic stroke care in Poland: analysis of claims database of National Health Fund in 2017. Neurol Neurochir Pol. 2020; 54(5): 449-455, doi: 10.5603/PJNNS.a2020.0066, indexed in Pubmed: 32885830.

23. Asuzu D, Nystrom K, Amin H, et al. Comparison of 8 scores for predicting symptomatic intracerebral hemorrhage after IV thrombolysis. Neurocrit Care. 2015; 22(2): 229-233, doi: 10.1007/s12028-0140060-2, indexed in Pubmed: 25168743.

24. Asuzu D, Nyström K, Amin H, et al. TURN: A Simple Predictor of Symptomatic Intracerebral Hemorrhage After IV Thrombolysis. Neurocrit Care. 2015; 23(2): 166-171, doi: 10.1007/s12028-015-0131-z, indexed in Pubmed: 25869481.

25. Asuzu D, Nyström K, Amin H, et al. Validation of TURN, a simple predictor of symptomatic intracerebral hemorrhage after IV thrombolysis. Clin Neurol Neurosurg. 2016; 146: 71-75, doi: 10.1016/j.clineuro.2016.04.017, indexed in Pubmed: 27152469. 
26. Kalinin MN, Khasanova DR, Ibatullin MM. The hemorrhagic transformation index score: a prediction tool in middle cerebral artery ischemic stroke. BMC Neurol. 2017; 17(1): 177, doi: 10.1186/s12883017-0958-3, indexed in Pubmed: 28882130.

27. Zhang J, Yang Yi, Sun $\mathrm{H}$, et al. Hemorrhagic transformation after cerebral infarction: current concepts and challenges. Ann Transl Med. 2014; 2(8): 81, doi: 10.3978/j.issn.2305-5839.2014.08.08, indexed in Pubmed: 25333056.

28. Spronk E, Sykes G, Falcione S, et al. Hemorrhagic Transformation in Ischemic Stroke and the Role of Inflammation. Front Neurol. 2021; 12: 661955, doi: 10.3389/fneur.2021.661955, indexed in Pubmed: 34054705.

29. Zhang S, Chen W, Tang H, et al. The Prognostic Value of a Four-Dimensional CT Angiography-Based Collateral Grading Scale for Reperfusion Therapy in Acute Ischemic Stroke Patients. PLoS One. 2016; 11(8): e0160502, doi: 10.1371/journal.pone.0160502, indexed in Pubmed: 27505435.

30. Hao $\mathrm{Y}$, Yang D, Wang $\mathrm{H}$, et al. ACTUAL Investigators (Endovascular Treatment for Acute Anterior Circulation Ischemic Stroke Registry). Predictors for Symptomatic Intracranial Hemorrhage After Endovascular Treatment of Acute Ischemic Stroke. Stroke. 2017; 48(5): 12031209, doi: 10.1161/STROKEAHA.116.016368, indexed in Pubmed: 28373302.
31. Nowak K, Derbisz J, Jagiełła J, et al. Time from stroke onset to groin puncture affects rate of recanalisation after mechanical thrombectomy: a real-life single centre experience. Neurol Neurochir Pol. 2020; 54(2): 156-160, doi: 10.5603/PJNNS.a2020.0024, indexed in Pubmed: 32242914.

32. Wang Bg, Yang N, Lin M, et al. Analysis of risk factors of hemorrhagic transformation after acute ischemic stroke: cerebral microbleeds do not correlate with hemorrhagic transformation. Cell Biochem Biophys. 2014; 70(1): 135-142, doi: 10.1007/s12013-014-9869-8, indexed in Pubmed: 24691925.

33. Sun F, Liu H, Fu HX, et al. Predictive Factors of Hemorrhage After Thrombolysis in Patients With Acute Ischemic Stroke. Front Neurol. 2020; 11: 551157, doi: 10.3389/fneur.2020.551157, indexed in Pubmed: 33224083.

34. Liu J, Wang Y, Jin Y, et al. Prediction of Hemorrhagic Transformation After Ischemic Stroke: Development and Validation Study of a Novel Multi-biomarker Model. Front Aging Neurosci. 2021; 13: 667934, doi: 10.3389/fnagi.2021.667934, indexed in Pubmed: 34122045.

35. Castellanos M, van Eendenburg C, Gubern C, et al. Low Levels of Caveolin-1 Predict Symptomatic Bleeding After Thrombolytic Therapy in Patients With Acute Ischemic Stroke. Stroke. 2018; 49(6): 15251527, doi: 10.1161/STROKEAHA.118.020683, indexed in Pubmed: 29712879. 